\title{
Battery capacity and recharging needs for electric buses in city transit service
}

\author{
Zhiming Gao, Zhenhong Lin, Tim J. LaClair, Changzheng Liu, Jan-Mou Li \\ Oak Ridge National Laboratory \\ P.O. Box 2008 \\ Oak Ridge, TN 37831
}

\author{
Alicia K. Birky \\ Energetics Incorporated \\ 7067 Columbia Gateway Dr, Suite 200 \\ Columbia, MD 21046 \\ Jacob Ward \\ Vehicle Technologies Office \\ Mailing Address: EE-3V \\ Room 5G-030 \\ 1000 Independence Ave, SW \\ Washington, DC 20585
}

\author{
Corresponding author: Zhiming Gao \\ Email address: gaoz@ornl.gov \\ Tel: +1-865-946-1339; Fax: +1-865-946-1354 \\ Oak Ridge National Laboratory \\ National Transportation Research Center \\ 2360 Cherahala Boulevard \\ Knoxville, TN 37932
}




\title{
Submitted to Energy -An International Journal
}

\begin{abstract}
This paper evaluates the energy consumption and battery performance of city transit electric buses operating on real day-to-day routes and standardized bus drive cycles, based on a developed framework tool that links bus electrification feasibility with real-world vehicle performance, city transit bus service reliability, battery sizing and charging infrastructure. The impacts of battery capacity combined with regular and ultrafast charging over different routes have been analyzed in terms of the ability to maintain city transit bus service reliability like conventional buses. The results show that ultrafast charging via frequent short-time boost charging events, for example at a designated bus stop after completing each circuit of an assigned route, can play a significant role in reducing the battery size and can eliminate the need for longer duration charging events that would cause schedule delays. The analysis presented shows that significant benefits can be realized by employing multiple battery configurations and flexible battery swapping practices in electric buses. These flexible design and use options will allow electric buses to service routes of varying city driving patterns and can therefore enable meaningful reductions to the cost of the vehicle and battery while ensuring service that is as reliable as conventional buses.
\end{abstract}

Key Words: Bus Electrification, Battery Sizing, Ultrafast Charging, City Transit Service 


\section{Submitted to Energy -An International Journal}

\section{Introduction}

Included among the seven million medium-duty (MD) vehicles currently registered in the U.S. are a significant number of city transit buses which receive significant federal, state and local subsidies [1-2]. Most city transit buses, which often are still powered by diesel engines, travel more than ten hours and one hundred miles daily. Despite being equipped with state-of-the-art aftertreatment devices to meet stringent emissions regulations, Gao et.al. [1] and Noel et.al [3] reported that these diesel buses contribute significantly to air pollution and related air-borne health issues in urban areas. Meanwhile, their frequent stop-and-go operation and significant idling time lead to poor fuel economy and generate remarkable amounts of greenhouse gases (GHGs), which lead to long-term effects on global warming. Unlike conventional vehicles, electric vehicles (EVs) offer zero direct tailpipe emissions, zero direct GHGs, and better energy efficiency as a result of braking energy recovery and no idling energy loss. Furthermore, there is potential for EVs to be the most energy efficient and sustainable choice for vehicle propulsion when it is coupled with renewable electricity use. Noel et.al [3] show that the concerns of fuel expenses, GHG health effects, and climate change encourage the use of electric buses in city transportation services.

Electric transit buses are being manufactured and operated in demonstration phases or pilot programs around the world, including China, Europe and North America [4-12]. Table 1 lists a variety of electric bus designs that are presently available from original equipment manufacturers (OEMs). The data indicate that battery and motor size, charger power capabilities and other electric powertrain design parameters for transit buses vary significantly among the OEMs. For example, the reported battery capacity varies from 60 to $548 \mathrm{kWh}$, with the most typical capacity levels in the 200-300 kWh range. Shuttle and trolleybuses usually adopt smaller battery capacity, as seen in Table 1. Meanwhile, there are at least two different ways of recharging electric buses, including on-route charging and overnight charging. The former is used to charge buses during vehicle operation while the latter charges buses at night or when the vehicle is not in operation. According to a report from the U.S. Federal Transit Administration (FTA) [4], on-route charging is typically done at a high rate of power (up to $400-500 \mathrm{~kW}$ ), whereas the overnight charging can be done at a lower power rate.

To support the implementation and market penetration of electric transit buses, substantial studies have been conducted to explore electric bus powertrains, EV operational ranges, cost-benefit analyses, and real-world operational assessments [13-21]. The research on electric bus powertrains includes the development of an electric bus with unique energy storage and/or other powertrain/drivetrain configurations [13-14], electric bus energy management [15-16], and regenerative braking ability [17]. These studies focus on detailed electric components and systems, and show electric powertrain improvements in energy efficiency. The EV operation range for battery size optimization has been highlighted in many studies. Lin [18] points out that appropriate battery size is crucial to both technical performance and economic viability because large battery packs represent a substantial cost of electric buses. The studies of cost-benefit analysis are dedicated to understanding the relationship between the vehicle price, battery cost and operational cost savings. Not surprisingly, the conclusions of these studies show that the vehicle cost and battery energy systems are some of the most critical factors for the adoption and use of EVs. Moreover, the life-cycle cost-benefits depend on operational routes and schedules, as reported by Nurhadi et.al.[19] and Lajunen [20]. In contrast to automotive EV powertrain technologies and cost-benefit analysis, examples of real-world operations of electric buses are still rather limited [21]. This is mainly due to a very limited number of electric buses available for fleet operations. Miles and his colleagues [22-23] studied the real-world performance of 8 electric buses running 17 hours per day along a 24-km (i.e. 15-mile) route. In the study, the buses were plugged in for overnight charging at the depot to fully charge the battery. During the day, they operated on the planned route and received $140 \mathrm{~kW}$ wireless boost charging at the start and end of the test route. This study showed that high power wireless charging could significantly extend the electric bus driving range. Zhou et.al. [24] also tested three electric buses on a 8.9-km (i.e. 5.5-mile) route and reported data covering $500 \mathrm{~km}$ (i.e. $310 \mathrm{miles}$ ) of testing. These limited studies were conducted only under relatively simple and limited test routes, which lack the complexity and variation of more extensive real-world driving conditions. The results do not fully reveal the impact of electric bus recharging activities and range limitations on maintaining consistent, periodic schedules for complex and varied routes without service interruption. Thus there are important gaps in current electric bus technologies for satisfying city transit bus needs which have not been well identified. This is apparently due to a lack of substantial data or studies focused on a comprehensive evaluation of electric buses in urban operations.

Since many transit buses operate in continuous service throughout the day along the planned route, the ability to maintain a periodic schedule on the route is very important. For an electric bus, if the energy remaining in the battery at the end of a circuit is below the level needed to complete the next circuit of the route (including an appropriate reserve), then it will be necessary to perform charging until this minimum level of energy is stored before driving the circuit. If an extended duration of charging is required, this could either result in a schedule delay or an additional bus may need to be dispatched to continue servicing the route while the original bus is recharged. Therefore, it is important to understand the impacts of electric bus charging needs on maintaining a consistent schedule, since "schedule reliability" of city transit bus service is a critical part of bus quality of service.

To address the issues identified above, a framework tool was developed as part of this study that links bus electrification feasibility with real-world vehicle performance, city transit bus service reliability, battery capacity sizing and charging infrastructure. The tool is used to evaluate the energy consumption and battery performance of city transit electric buses using extensive drive cycle data 


\section{Submitted to Energy -An International Journal}

measured from several buses during one year of operation in a city transit bus fleet, so the data are very representative of real-world routes driven by conventional diesel buses. Several standardized bus drive cycles are also evaluated for comparison. The emphasis is to understand how the selection of appropriate battery capacity and recharging options can minimize limitations associated with bus electrification and to identify electric bus applications and battery design choices that are most suitable for complex city transportation requirements. To keep the problem tractable, the analysis of energy consumption was simplified by using a vehicle tractive energy method and component efficiency models for describing the bus system performance as opposed to developing detailed component and system models. Appropriate experimental data and commercial software are also employed to validate the method.

\section{Method, Driving Data and Vehicle Assumptions}

A framework tool was developed to link bus electrification feasibility with real-world vehicle performance, city transit service reliability, battery sizing and charging infrastructure. The general structure for this framework is shown in Figure 1. According to the selected bus and its related drive-cycle database, the driving behavior and road conditions are first identified, and Autonomie software is used to determine the system/component efficiencies and key parameters of the simulated vehicle over typical driving conditions. Autonomie is an open-architecture powertrain and vehicle systems simulation tool developed by Argonne National Laboratory [2526]. Then a tractive energy model including the identified system/component efficiencies and key parameters is used to evaluate the energy consumption and battery performance of transit electric buses over various real-world city routes. In the studies of energy consumption and battery performance, parameters characterizing the transportation and charging infrastructure, battery sizing properties, and battery operating threshold are included as inputs for the EV and battery performance analysis. The tractive energy model can be calibrated using experimental data and/or results from commercial software. The tool allows the user to evaluate the EV performance, optimize battery capacity and charging infrastructure options, and identify opportunities and gaps associated with vehicle electrification on real-world transit bus service reliability. The model was designed to be flexible, allowing the selection of a variety of battery technology data, charging schedules, and different routes, and it can be used to evaluate the electrification potential for a range of vehicle types including light-, medium- and heavy-duty vehicles. To the authors' knowledge, no other such tool to assess vehicle electrification is available in the public domain.

The next section describes the main functionality and data used in the tool, including details used for the tractive energy, regenerative braking, and vehicle component efficiencies, as well as drive cycle data and vehicle component assumptions for both conventional and electric transit buses.

\subsection{Vehicle Energy Calculation}

A common approach using the tractive power, as reported by LaClair et.al. [27] and Gao et.al. [28], is adopted to determine the power requirement of the vehicle powertrain based on the transit bus's forward speed, acceleration, rotational inertia, aerodynamic loss, rolling resistance loss, and the road grade. For any instant of time, the bus tractive power is described as:

$W_{\text {tract }}=m \cdot V \cdot d V / d t+\frac{1}{2} \rho \cdot C_{d} \cdot A_{f} \cdot V^{3}+m \cdot g \cdot C_{r r} \cdot V+m \cdot g \cdot V \cdot \sin (\theta)$

where $W_{\text {tract }}$ is the tractive power of the bus; $V$ is velocity; $\rho$ is air density; $C_{d}$ is the bus's aerodynamic drag coefficient; $C_{r r}$ is the rolling resistance coefficient; $A_{f}$ is the frontal area; $\theta$ is road grade; $g$ is the gravitational acceleration constant; $t$ is operating time; and $m$ is the total bus mass, including passengers. The model accounts for mass of many key components for the vehicle powertrain and drivetrain systems (e.g., engine, clutch/torque, gearbox, final drive, wheel, chassis, generator, battery, mechanical and electrical accessory, as well as motor and high-voltage battery for hybrid powertrain).

For a conventional vehicle, the tractive power is positive when the vehicle is being actively propelled and the engine provides power to the wheels. However, the tractive force becomes negative during periods of braking. Braking represents a dissipative force that depletes the energy that is effectively stored as vehicle kinetic and potential energy. Tractive power becomes zero when the vehicle is idling, but the engine still has to run to fulfill accessory loads. Accounting for the drivetrain component efficiencies, the mechanical power output from the engine, $W_{\text {eng }}^{c}$, can be calculated as shown below:

$$
\begin{array}{lll}
\text { Powered driving: } & W_{\text {eng }}^{c}=W_{\text {tract }}^{c} / \eta_{w h} \cdot \eta_{f d} \cdot \eta_{g b} \cdot \eta_{c l}+W_{a c c}^{c} & \forall\left(W_{\text {tract }}^{c}>0\right) \\
\text { Braking or idle: } & W_{\text {eng }}^{c}=W_{a c c}^{c} & \forall\left(W_{\text {tract }}^{c} \leq 0\right) \text { : }
\end{array}
$$

Where $\eta_{w h}$ is wheel efficiency; $\eta_{f d}$ is final drive efficiency; $\eta_{g b}$ is gearbox efficiency; $\eta_{c l}$ is clutch efficiency; and $W_{a c c}^{c}$ is accessory load of a conventional vehicle. The conventional bus fuel consumption is given as

$\dot{m}_{f}=W_{e n g}^{c} / \eta_{\text {eng }} \cdot L H V_{f}$ 


\section{Submitted to Energy -An International Journal}

where $\dot{m}_{f}$ is the fuel consumption rate. The engine efficiency is taken to be a function of the engine power, as follows:

$\eta_{\text {eng }}=1.172 \cdot\left(W_{\text {eng }}^{c} / W_{\text {eng }}^{p}\right)^{0.5}-1.006 \cdot\left(W_{\text {eng }}^{c} / W_{\text {eng }}^{p}\right)+0.231 \cdot\left(W_{\text {eng }}^{c} / W_{\text {eng }}^{p}\right)^{2}$

In this equation, $L H V_{f}$ is the fuel lower heating value (43,500 kJ/kg for diesel); $W_{e n g}^{p}$ is the engine peak power; and $\eta_{\text {eng }}$ is the engine efficiency. Equation (3b) was derived based on a MD engine map [1].

For an electric vehicle, electric power output from the battery is estimated based on the efficiencies of the electric components (i.e., motor and battery) and related drivetrain components (e.g., final drive and wheel), as shown in Eq. (4). Unlike a conventional vehicle, an EV is capable of converting vehicle kinetic energy into a storable form of battery energy during braking if the thresholds of vehicle speed and acceleration are satisfied. This EV model assumes that energy regeneration from braking occurs as long as the vehicle acceleration does not exceed a threshold level and the vehicle speed has not fallen below a specified value, as described in Eq. 4. The constraints are used in order to distinguish rapid braking events (such as during vehicle emergency braking) when regeneration is not possible from kinetic energy regeneration, and to avoid very low kinetic energy regeneration.

For electric transit buses, it is important to consider the specific charging activities carried out, particularly in aggressive daily operations. In fact, the effectiveness of EV charging substantially depends on the vehicle's battery capacity and the power available from the EV charging stations. Compared to EV chargers used for passenger cars, commercial vehicle chargers, including inductive charging and conductive charging, are capable of providing considerably greater power. Usually inductive charging yields a relatively low power transfer rate of 50-200 kW, while conductive charging provides a higher power transfer rate of $300 \mathrm{~kW}$ and up [29]. The reported maximum power transfer with conductive charging is $500 \mathrm{~kW}$ [7]. To understand the impacts of different charging power levels, inductive or conductive charging technologies are allowed to service both on-route and overnight charging in the model. The charging efficiency is also assumed to be constant.

$\begin{array}{lll}\text { Powered driving: } & W_{\text {dis }}^{e}=W_{a c c}^{e}+W_{\text {tract }}^{e} / \eta_{w h} \cdot \eta_{f d} \cdot \eta_{\text {mot }} \cdot \eta_{\text {batt }} & \forall\left(W_{\text {tract }}^{c}>0\right) \\ \text { Braking wo regen: } & W_{\text {dis }}^{e}=W_{\text {acc }}^{e} & \forall\left(W_{\text {tract }}^{e}<0\right) \cap\left\{\left(a>a_{h b}\right) \cup\left(V<V_{l b}\right)\right\} \\ \text { Braking w/ regen: } & W_{c h g}^{e}=-W_{a c c}^{e}+\left|W_{\text {tract }}^{e}\right| / \eta_{w h} \cdot \eta_{f d} \cdot \eta_{\text {mot }} \cdot \eta_{\text {batt }} & \forall\left(W_{\text {tract }}^{e}<0\right) \cap\left(a<a_{h b}\right) \cap\left(V>V_{l b}\right) \\ \text { Idle: } & W_{\text {dis }}^{e}=W_{a c c}^{e} & \forall\left(W_{\text {tract }}^{e}=0\right) \\ \text { Recharging event: } & W_{c h g}^{e}=W_{\text {orc }}^{e} \cdot \eta_{\text {chgr }} \text { or } W_{n c}^{e} \cdot \eta_{c h g r} & \forall\left(W_{\text {tract }}^{e}=0\right) \cap\left(\vec{X} \in \vec{C}_{i}\right)\end{array}$

where $\eta_{m o t}$ is the motor efficiency; $\eta_{\text {batt }}$ is battery efficiency based on impedance; $\eta_{c h g r}$ is the charger efficiency; $a$ is acceleration; $a_{h l}$ (i.e. $a_{h b}=-3.0 \mathrm{~m} / \mathrm{s}^{2}$ ) is the maximum acceleration (high bound) for which the regenerative braking system can convert kinetic energy to electric energy; $V_{l b}$ (i.e. $V_{l b}=5.0 \mathrm{~m} / \mathrm{s}$ ) is lower bound for speed in converting kinetic energy into electric energy; $W_{a c c}^{e}$ is the accessory load of the electric vehicle; $W_{d i s}^{e}$ is the battery discharge power; $W_{c h}^{e}$ is the battery charge power from regenerated kinetic energy; $W_{\text {orc }}^{e}$ is the on-route charging power; $W_{n c}^{e}$ is the night-time charging power; $\vec{C}_{i}$ is the $i^{\text {th }}$ charger location; and $\vec{X}$ is the vehicle location. Therefore, the battery state of charge (SOC) can be described as

$S O C=1-\int_{0}^{t}\left(W_{d i s}^{e}-W_{c h g}^{e}\right) d t / E_{\text {batt }}$

where $E_{\text {batt }}$ is the battery's energy storage capacity.

Finally, a simulation tool was developed in the Matlab programming environment based on this tractive energy-component efficiency approach to calculate the component and system performance. The user enters driving data, vehicle component efficiency, vehicle weight, battery properties, charging locations and charger power data into a designated Microsoft Excel format, and the tool performs calculations to quantify the impact of the battery capacity and charger use on energy consumption and the transit bus service reliability.

\subsection{Driving Data}

To reflect extensive real-world bus driving conditions, the Oak Ridge National Laboratory (ORNL) MD conventional bus database [30] was used. The database covers one year of real-world second-by-second measurements from three Class-7 diesel buses operated by the Knoxville Area Transit (KAT) in Knoxville, TN. Knoxville is a typical U.S. medium-sized city with nearly 300,000 residents. The studied KAT buses were all 2005 Optima LF-34 models. Each bus ran along four major routes throughout the Knoxville area (see Figure 2) on either single or multiple routes all day. Plus, some of the designated routes change with the time of year and even between morning and afternoon schedules. Figure 2 shows the complexity of city transit bus operations, with frequent variations of scheduled bus services and different ranges for the routes. The typical daily operation for the KAT bus started at the garage where all 


\section{Submitted to Energy -An International Journal}

the buses return at the end of daily operations. At the start of each day, the KAT buses travel to the bus depot before beginning daily services, and the depot also serves as both the starting point and ending point for each route (see Figure 2, where the bus depot and garage are shown with a red and blue dot, respectively). Although most of the routes traveled by the KAT buses involved short trips on city streets, highway data was also collected. The data were recorded for 610 days, covering 4,714 hours (including 3,287 route hours of travel) and 71,479 km (i.e. 44,397 miles). Figure 3 shows driving performance statistics for the three KAT buses. Table 2 lists several key driving characteristics for this measured data, which is referred to as the KAT cycle.

The real-world second-by-second data were collected using seventy-three signals from a suite of deployed sensors and various vehicle system data obtained from each vehicle's SAE J1939 and J1708 data bus [30]. The key measured data included fuel consumption, vehicle speed and acceleration, engine speed and torque, vehicle weight, and global positioning system (GPS) spatial location information. The data for fuel consumption, speed, transient weight with passenger loading, and GPS location were used in the evaluation of tractive power and the coefficients used in Eqs. 1-5. For example, the GPS altitude data were used to estimate road grade, $\theta$, which can have a significant impact on vehicle powertrain performance and energy consumption (see Eq. 1). Because roads are typically built as a set of segments of constant slope connected by well-defined curves, the grade of the road traveled by a vehicle participating in the study was computed using the following approach: initial road grade for 30-meter (i.e. 100 feet) travel segments was generated based on distance and GPS altitude profiles; this procedure was conducted for all segments until all of the recorded data was processed. This method was found to function well, providing consistent information for the roadway grade over the repeated segments, as reported by Lascurain et al. [30]. The GPS location information for the bus garage and depot were also used to designate the charging locations.

Since different city transit buses experience different road and traffic conditions, diverse bus driving cycles have been developed [1]. To represent a broader range of practices, four separate drive cycles covering average cycle speeds ranging from 5.75 to $19.85 \mathrm{~km} / \mathrm{hr}$ (i.e. 3.57-12.33 mph) were also selected for the analysis (see Table 2). These standardized drive cycles include the Manhattan bus cycle (MAN), the New York bus cycle (NYBC), the Orange County Transit Authority bus cycle (OCTA), and the Washington Metropolitan Area Transit Authority cycle (WMATA). The standardized drive cycles do not include road grade. Compared to these cycles, the real KAT drive-cycle data show a considerably higher maximum speed (due to routes including interstate highway travel), while the KAT data for average speed and idle time are still relatively close to the other city bus cycle data. This reflects the complexity of real city driving conditions (including real grade and highway travel) which should not be ignored and are important for evaluating electric buses operating under urban conditions.

\subsection{Vehicle and Charging Assumptions}

A conventional transit bus configuration was first constructed based on the 2005 Optima LF-34 bus, which was characterized in the ORNL MD conventional bus database. Table 3 lists the specifications of the modeled conventional transit bus, where the frontal area, rolling resistance, and aerodynamic drag coefficients were estimated and corrected using Autonomie software based on fuel consumption and engine power measurements from the ORNL MD conventional bus database. The appropriate constant (average) efficiencies for each component were derived from Autonomie simulations, and a constant value was also adopted for power consumption relative to all accessories. The constant load does not account for complex bus space heating and cooling loads which vary with the seasons and time of a day.

To confirm that the assumptions gave reasonable simulation results for a conventional bus, the 2005 Optima simulations were compared with two-hours of measured data selected from the full set of real-world KAT bus data [1, 30]. For the two-hour data with a travel distance of $46.05 \mathrm{~km}$ (i.e. 28.6 miles) (see Figure 4), the predicted and measured trip engine output powers were $86.8 \mathrm{kWh}$ and $85.8 \mathrm{kWh}$, respectively. Autonomie was also employed to simulate the same scenario. The prediction by Autonomie is $89.4 \mathrm{kWh}$ which is close to the simulated result by the current method. The predicted diesel fuel consumption has less than $4 \%$ error when compared to the measurement. Figure 4(a) shows a good agreement between the simulations and measurements for engine energy and fuel consumption, implying that the basic simulation assumptions for the conventional powertrain were indeed reasonable. In the validation, the vehicle weight was $11,636 \mathrm{~kg}$.

For the electric bus simulations, the major vehicle components considered were the battery, motor, final drive, wheel and chassis. Again, the authors assumed constant average efficiencies for motor and battery components. Table 3 summarizes these characteristics for the main electric vehicle systems. Other drivetrain components and chassis parameters remained the same as those used in the conventional bus. Similarly, a constant value for the accessory load is used in the electric bus simulation. Since there are less accessory components in an EV, the accessory load for the electric bus is assumed to be $50 \%$ less than that used in a conventional vehicle based on data published in the literature [32-34]. However, compared to a conventional vehicle, the battery and related electronic components used in an electric vehicle typically lead to a significant weight penalty due to the low energy density of the battery. Thus the EV weight penalty was estimated as

$m_{\text {penalty }}=m_{\text {batt }}+m_{m o t}-m_{\text {eng }}-m_{g b}$ 


\section{Submitted to Energy -An International Journal}

where $m_{b a t t}, m_{m o t}, m_{e n g}$, and $m_{g b}$ are the weight of battery, motor, engine and gearbox, respectively. Their values are estimated based on the component peak powers except for the battery which is estimated based on the battery capacity. The detailed mass estimations are considered as $m_{\text {batt }}=6.67 \cdot E_{\text {batt }}$ (based on Li-ion battery), $m_{\text {mot }}=1.20 \cdot W_{\text {mot }}^{P}, m_{\text {eng }}=1.55 \cdot W_{\text {eng }}^{P}$, and $m_{g b}=$ $0.50 \cdot W_{g b}^{P}$, for power values in units of $\mathrm{kW}$. The coefficients used were obtained from references [35-37]. The variables, $W_{m o t}^{P}, W_{e n g}^{P}$, and $W_{g b}^{P}$, represent the peak power for motor, engine and gearbox, respectively. In the current research, all of these are defined as $200 \mathrm{~kW}$ in accordance with the 2005 Optima LF-34 bus.

Since there is no data available for electric buses, it is difficult to calibrate the electric bus model directly. To confirm the above assumptions give reasonable electric bus predictions, Autonomie software was used to construct a virtual electric bus derived from the 2005 Optima bus configuration but with the EV specifications listed in Table 3. The Autonomie-predicted battery energy consumption was then compared with the current model for the same drive cycle scenario used in Figure 4(a) indicating a $2 \%$ error only. Figure 4(b) shows good agreement between the simulation using the current model and the prediction using Autonomie, implying that these electric bus assumptions are reasonable. In the electric bus validation, the vehicle weight is $13,626 \mathrm{~kg}$. The electric vehicle weight penalty is $1,990 \mathrm{~kg}$ based on Eq. 6 .

To reflect real situations for all-electric city buses, EV charging requirements were considered in the simulations. Three charging modes are considered: boost charging, proactive charging (defined below) and overnight charging. The boost charging and proactive charging are considered as on-route charging events. The assumptions include (1) boost charging which occurs when the bus stops briefly at the depot after finishing each loop of operation; (2) proactive charging is considered to take place at the depot if a specific charging event with an extended period is required (along with skipping of the next one or more circuits of the route, indicating that the transit bus service reliability criterion has been violated); (3) overnight charging at the bus garage; and (4) the range of charging power employed in the simulations is $90 \mathrm{~kW}$ to $480 \mathrm{~kW}$, and the charger efficiency is $97 \%$ [31]. The charger efficiency is higher than $91 \%$ as recommended by Tesla, which is used for passenger cars [38]. The reason is that the charger voltage for commercial vehicle EV charging is $600 \mathrm{~V}-750 \mathrm{~V}$, while the voltage for charging of passenger cars is $120 \mathrm{~V}-240 \mathrm{~V}$. The higher voltage is expected to provide a benefit in reduced electrical energy losses. In the simulations, overnight charging is defined as charging that occurs during the period from when buses end daily operation and return to the bus garage until buses start daily operation the next day. To simplify overnight charging, the simulated buses are assumed to be fully charged each morning. The boost charging occurs during regular short stops at the bus depot after finishing every circuit of the planned route; the time is based on bus driving data (typically around 5-10 minutes in Knoxville). The proactive charging only occurs if the stored battery energy following the assumed level of boost charging is insufficient for powering the bus through the next circuit of the route. In determining the stored battery energy, to avoid having too low of a SOC level, a low threshold for SOC is established to secure battery reliability and performance stability. The threshold for low SOC assumed for this model is $10 \%$. Once the current SOC reaches this level or the predicted SOC during the next circuit will surpass this threshold, the proactive charging activity is then required, and the battery is charged with the minimum energy or time by skipping one or more circuits along the route. Following this strategy, the battery SOC will never fall below $10 \%$, so the battery is protected from excessive discharging and the bus will not be stranded due to a fully depleted battery.

\section{Results and Discussions}

In this section, the simulated energy consumption and charging activities are presented using real-world data from the KAT drive cycles and the four standardized bus drive cycles; then the impact of boost charging and proactive charging is discussed with respect to the electric bus range, battery capacity and charging, and transit bus service reliability.

\subsection{Energy consumption and charging activities}

Figure 5(a) compares the cumulative daily mechanical energy consumption by the conventional vehicle engine with the net electrical energy consumed by the electric bus battery over the KAT real-world driving conditions. The impact of passenger loading is considered in the analysis, using the passenger loading data available from the ORNL MD conventional bus database. In the electric bus simulation using a $324 \mathrm{kWh}$ battery and EV charger power of $90 \mathrm{~kW}$, the minimum proactive charging time is 2 hours. The average battery energy consumption of the electric bus is $1.35 \mathrm{kWh} / \mathrm{km}$ (i.e. $2.17 \mathrm{kWh} / \mathrm{mile}$ ), while for the conventional vehicle case the average mechanical energy consumption by the engine is $1.80 \mathrm{kWh} / \mathrm{km}$ (i.e. $2.89 \mathrm{kWh} / \mathrm{mile}$ ). The corresponding diesel fuel energy consumption is $5.52 \mathrm{kWh} / \mathrm{km}$ (i.e. $8.89 \mathrm{kWh} / \mathrm{mile}$ ) based on an engine efficiency of $32.5 \%$. Clearly bus electrification improves vehicle energy saving. In the simulated electric bus, the average braking energy recovered is nearly $0.39 \mathrm{kWh} / \mathrm{km}$ (i.e. 0.63 $\mathrm{kWh} / \mathrm{mile}$ ), without which the battery energy consumption would be $29 \%$ greater. Figure 5 (b) shows details of the electrical energy recovered from kinetic energy by the braking regeneration system. However, the overall enabled driving distance by the simulated electric buses is $66,461 \mathrm{~km}$ (i.e. 41,280 miles) which is $7 \%$ less than conventional diesel buses due to skipping of some circuits of the route as a result of the proactive charging. 


\section{Submitted to Energy -An International Journal}

Figure 6 shows the proactive charging demand as a function of daily drive mileage and time. The results show 87 days of the total 610 drive days need proactive charging, meaning $14.2 \%$ of the overall drive days. Most of these days are typically operating more than 12 hours and $322 \mathrm{~km}$ (i.e. 200 mile) per day. For the simulated battery size of $324 \mathrm{kWh}$, the limitation of 10-hour drive and $242 \mathrm{~km}$ (i.e. 150 miles) per day could be a critical threshold for scheduling transit bus daily operation under drive conditions like Knoxville, TN, US in the absence of any on-route recharging facility.

Reducing battery capacity is particularly attractive for electric vehicle cost reduction, since the battery cost is a major portion of electric vehicle life cost. Not surprisingly, electric bus performance changes significantly with different battery sizes, as shown in Figure 7. With a decrease of the battery size from $324 \mathrm{kWh}$ to $150 \mathrm{kWh}$, the proactive charging days increases up to 208 days of the overall 610 days, and the lost drive time is 971 hours, which is nearly $30 \%$ of the total 3287 route driving hours. This means significant additional bus purchases would be necessary to make up the bus service lost due to EV battery range limitations. Evidently, it is not an optimal to reduce battery size.

Figure 8 provides two examples of how battery size and bus route range alter proactive charging activities and battery SOC performance. One is a short route with $20.9 \mathrm{~km}$ (i.e. 13 miles) and 1-hour circuit time, and the other is a longer route with $61.2 \mathrm{~km}$ (i.e. 38 miles) and 2.5-hour circuit time. In both routes, transit buses stop at the depot for about ten minutes or less after finishing each circuit operation. In the simulation, boost charging is assumed to occur during the short stop at the bus depot. The results show that both a $150 \mathrm{kWh}$ and $324 \mathrm{kWh}$ battery require proactive recharging in addition to the regular boost charging. The short boost charging with $90 \mathrm{~kW}$ does not play a significant role although very limited "boost waves" are found in Figure 8 . The data also indicate that the smaller battery capacity leads to a higher frequency of proactive recharging. Moreover, compared to a $324 \mathrm{kWh}$ battery, a $150 \mathrm{kWh}$ battery leads to more frequent recharging and missed circuit operations when following a longer route (see Figure 8(b)). In the figure, the red dash rectangles are for $150 \mathrm{kWh}$ batteries and the green solid-line rectangles are for $324 \mathrm{kWh}$ batteries for the proactive charging activities (or missed circuit operation), respectively.

To better understand the impact of battery capacity on different city transportation services, the study was further conducted over the four standardized drive cycles: MAN, NYBC, OCTA and WMATA. These cycles reflect different city bus operations, but do not account for real route information such as bus depot and garage characteristics. Thus, the simulations did not include any recharge activities. Instead it is simply assumed that simulated buses operating with various battery capacities operate continuously along the given drive cycles until the battery energy hits the protected 10\% SOC for battery reliability and safety. The results shown in Figure 9 indicate that the simulated electric energy consumption per unit mileage varies from $1.24 \sim 2.48 \mathrm{kWh} / \mathrm{km}$ (i.e. $2-4 \mathrm{kWh} / \mathrm{miles}$ ), which is significantly less than the engine mechanical energy of a conventional bus. Interestingly, the lower average cycle speed leads to higher electric energy consumption per km for both conventional diesel-powered and battery-powered buses (see Figure 9(a)). This is reasonable because a cycle with lower average speed usually has longer idle times, more braking loss, and a larger relative contribution to total energy from accessory loads like HVAC etc. On the other hand, Figures 9(b) and 9(c) indicate that a cycle with lower average cycle speed requires smaller battery sizes to meet daily service. For example, if considering 16-hours as maximum daily drive cycle, a $250 \mathrm{kWh}$ battery supports an 18-hour uninterrupted service for the NYBC cycle, but only allows for 10-hours of service for the OCTA cycle. The major reason is that lower average cycle speed, such as in the NYBC cycle, generates a shorter distance and consumes lower total energy in spite of the higher energy consumption per $\mathrm{km}$. Consequently, it is important for different city transportation authorities to choose the appropriate bus battery capacity, depending on the details of their particular transportation services, in order to achieve the greatest cost reduction. For the simulations of the four standardized drive cycles, the constant bus weight was considered to be $11,636 \mathrm{~kg}$ for the conventional bus and 12,526-13,626 kg for electric buses with $150-324 \mathrm{kWh}$ battery capacity.

\subsection{Impact of ultrafast charging}

As mentioned above, it is important to make an electric bus with flexible battery sizes in an effort to reduce cost. However, varying driving and route characteristics are observed within every city. Therefore, it is interesting to understand the impact of battery size, particularly together with appropriate emerging charging technologies (such as ultrafast charging) on an electric bus application along short and long routes. Ultrafast charging technology can run with $700 \mathrm{~V}$ to $800 \mathrm{~V}$ direct current (DC) to provide $400-500 \mathrm{~kW}$ output charging power with $>97 \%$ efficiency, which is higher than regular charging efficiency [31]. Figure 10 compares the impact of regular $90 \mathrm{~kW}$ charging and $480 \mathrm{~kW}$ ultrafast charging on proactive charging activities and missed drive time using the KAT real-world driving data. Compared to the $90 \mathrm{~kW}$ regular charging, ultrafast charging can significantly minimize the proactive charging demand and reduce the need to miss a number of circuits on the planned route. The impact becomes even more significant for a larger battery. For example, the proactive charging activities are decreased to zero with $324 \mathrm{kWh}$ battery combined with $480 \mathrm{~kW}$ ultrafast charging, meaning that the electric bus equipped with such a battery and ultrafast charging is cable of operating completely like a conventional diesel bus without any change to the transit buse service reliability.

Unlike the case of $90 \mathrm{~kW}$ boost charging, a high-power ultrafast charging, which can provide relatively significant levels of additional energy to the battery during even 5 to 10 minutes stops at the bus depot, can play a critical role in eliminating proactive charging events. Figure 11 shows how the boost charging changes the operation for a bus over both the short and long routes analyzed in Figure 


\section{Submitted to Energy -An International Journal}

8. For the short route, just 5 to 10 minutes of the $480 \mathrm{~kW}$ ultrafast charging can supply sufficient electrical energy to cover the entire route. Consequently, the bus battery size can be significantly reduced. Figure 11(a) shows that a $60 \mathrm{kWh}$ battery is adequate to power the simulated bus along the short route. This confirms findings of Miles and Kontou [23] that ultrafast charging technology can enable electric buses to provide transit bus service reliability on short city routes that is very consistent with the service reliability of a conventional diesel bus. For the long route, the $480 \mathrm{kWh}$ ultrafast charging technology allows the bus to reduce its battery capacity from $324 \mathrm{kWh}$ to $200 \mathrm{kWh}$ while fully eliminating the proactive charging through frequent short-term boost charging (see Figure 11(b)). Thus it is seen that high power ultrafast charging can also play a significant role in the case of longer routes that occur in real transit service operations, although it would not be possible in this case to reduce battery capacity by a significant degree.

The above results provide important insight on cost reductions that may be possible for the electric bus and battery. Among different cities, or even for different routes within the same city, very different driving characteristics may be experienced. Thus an optimal strategy for a fleet would be to have electric buses that are capable of using multiple battery capacity configurations and to use flexible battery swapping procedures based on the daily or weekly demand of the scheduled services. In other words, it would be ideal if electric buses are able to be frequently modified with different battery capacities based on the particular service needs. Each change to the battery capacity would be determined based on the driving range needed for the bus's planned service, instead of the buses themselves. This is particularly true with high power ultrafast charging technologies, which provide groundbreaking opportunities to reduce battery size in servicing both short and long routes. If EV OEMs can produce electric buses that permit multiple capacity configurations and easy battery swapping, the fleets only need to select and manage their battery components for electric buses based on their scheduled routes. Such electric buses would be capable of servicing any route in any city by having the appropriate battery capacity and following appropriate battery swapping practices as outlined above. In this manner, the cost of typical electric bus and battery could be significantly reduced because the overall battery capacity owned by each fleet would be reduced to a level that is tailored specifically to the fleet's actual operations. Furthermore, the batteries available within the fleet could be used in a flexible manner in any of its electric vehicles. This flexibility in design and battery usage can yield very significant cost benefits to the electric bus market.

Another important consideration is the impact of ultrafast charging on battery degradation and life. This is a rather complex issue since battery life depends strongly on stresses related to the thermal environment of the battery, anode/cathode chemistry, cell design and charging characteristics like the $\triangle$ SOC charging window [39]. The generally accepted theory is that ultrafast charging will increase the rate of battery degradation [39-40]. However, preliminary real-world applications [41-42] show that it is less damaging than previously believed. A study conducted at the Idaho National Laboratory (INL) [41] shows that while ultrafast charging of batteries does increase battery degradation compared to regular AC level $2 \mathrm{EV}$ charging, the difference in capacity loss associated with fast charging is significantly less than the overall loss of capacity experienced during the normal life of the battery. In the study, two Nissan Leafs with a $24 \mathrm{kWh}$ battery capacity were charged using $3.3 \mathrm{~kW}$ AC Level 2 chargers while two identical Leafs were charged using $50 \mathrm{~kW}$ DC fast chargers. In the latter case, the ratio of battery capacity to charger power-corresponding to the time that would be necessary to charge a battery from 0 to $100 \%$ SOC at full power-is $24 \mathrm{kWh} / 50 \mathrm{~kW}=0.48$ hour, which is faster than the charging time of 0.675 hour for a $324 \mathrm{kWh}$ battery / 480kW charger. After 20,000 miles of driving, the ultrafast charged vehicles had lost $8.4 \%$ of their initial battery capacity while the Level 2 charged vehicles had lost $8.2 \%$ of the initial capacity. After 50,000 miles of use, the fast charged vehicles had lost nearly $25 \%$ of the initial capacity while the Level 2 charged vehicles lost $22 \%$. These results indicate that both miles traveled and recharging activities degrade EV battery capacity and range, but the type of recharging appears less crucial than the miles traveled. This conclusion was confirmed in a second INL investigation [42]. Thus the concern that fast charging leads to severe battery performance degradation may be less significant than commonly believed, and there may be approaches that can be followed to further minimize any detrimental effects of rapid charging, Nonetheless, additional research is clearly needed to better understand the impacts of ultrafast charging on battery life and further characterize results in real-world applications.

\section{Conclusion}

A framework tool was developed to link bus electrification feasibility with real-world vehicle performance, city bus transit service reliability, battery sizing and charging infrastructure. It allows the user to evaluate the EV performance, optimize battery sizing and options for charging infrastructure, and identify opportunities and gaps associated with vehicle electrification in real-world city bus transit service. This tool can flexibly adopt various battery technology data, charging schedules, and different routes. The tool has been used in the present study to investigate an electric bus application to characterize the transit bus service reliability while following a range of scheduled routes by considering different options for battery configurations that could be used in the fleet as well as considering high power ultrafast charging of the buses. This analysis was performed using data corresponding to real day-to-day routes driven by conventional diesel buses in the KAT bus fleet as well as with standardized bus drive cycles.

For the KAT real-world driving conditions, the average battery energy consumption of the electric bus is $1.35 \mathrm{kWh} / \mathrm{km}(2.17$ $\mathrm{kWh} / \mathrm{mile})$, while for the conventional vehicle case the average mechanical energy consumption by the engine is $1.80 \mathrm{kWh} / \mathrm{km}$ ( 2.89 $\mathrm{kWh} / \mathrm{mile}$ ). These average levels of energy consumption are actually within the range of energy consumption calculated for the 


\section{Submitted to Energy -An International Journal}

standardized bus drive cycles For the standardized bus drive cycles the calculated electric bus energy consumption varies from 1.24 to $2.48 \mathrm{kWh} / \mathrm{km}(2.0-4.0 \mathrm{kWh} / \mathrm{mile})$ compared to $1.7 \sim 3.3 \mathrm{kWh} / \mathrm{km}(2.7-5.3 \mathrm{kWh} / \mathrm{mile})$ of mechanical energy required by the conventional diesel bus. The results of the analysis show that regenerative braking energy recovery plays a significant role in the electric bus energy savings. For example, for the full KAT real-world driving data, regenerative braking was found to reduce the battery energy consumption by approximately $29 \%$ relative to what would be required without regenerative braking. Although an economic analysis was not conducted in the present investigation, the energy savings during electric bus operations can be expected to offset at least some of the costs associated with the purchase of electric buses and potential battery replacement costs.

The use of high power ultrafast charging allows frequent and short-duration boost charging events which play a critical role in reducing the battery capacity needed for both short and long routes, while also eliminating the need for proactive charging events and avoiding service interruptions or delays due to required charging. The ultrafast charging can thus enable consistent transit bus service reliability in electric bus operations that is consistent with that of a conventional diesel bus. Real-world applications show that ultrafast charging does increase battery degradation, but the magnitude of capacity loss due to ultrafast charging appears less significant than that resulting from normal repeated charging over time. The concern that ultrafast charging will severely degrade battery performance may therefore be unfounded.

Considering the large variations in driving characteristics for different cities and individual bus routes, the current analysis shows that significant cost savings would be possible if transit bus fleets are able to employ electric buses capable of using multiple battery capacity configurations in addition to flexible battery swapping practices based on scheduled routes. The current practice of using electric bus with a large battery capacity to satisfy any routes or small battery capacity to serve only specific short routes results in a loss of operational flexibility, and very frequently excessive battery capacity will be deployed, resulting in excessive costs for the bus fleets. The flexibility in the bus battery selection and alternative charging practices for electric buses as proposed in this research would enable servicing any route with appropriate battery configurations. Furthermore, the proposed flexible battery swapping practices will enable fleets to manage and maintain the bus batteries to ensure appropriate battery capacity even for variable service needs. Overall, the cost of the vehicle and battery can be reduced with this approach while satisfying the fleet's service needs.

\section{Acknowledgement}

This project was sponsored by the US. Department of Energy's VTO program. The authors would like to recognize Oscar Franzese for his assistance in data analysis and Moore Sheila for her assistance in the paper preparation. We also appreciate the editors and reviewers for their time and support.

Notice: this manuscript has been authored by UT-Battelle, LLC under Contract No. DE-AC05-00OR22725 with the U.S. Department of Energy. The United States Government retains and the publisher, by accepting the article for publication, acknowledges that the United States Government retains a non-exclusive, paid-up, irrevocable, world-wide license to publish or reproduce the published form of this manuscript, or allow others to do so, for United States Government purposes. The Department of Energy will provide public access to these results of federally sponsored research in accordance with the DOE Public Access Plan.

$\begin{array}{ll}\text { Nomenclature } \\ A & : \text { Area } \mathrm{m}^{2} \\ a & : \text { Acceleration, } \mathrm{m} / \mathrm{s}^{2} \\ C & : \text { Coefficient } \\ \vec{C}_{i} & : i^{\text {th }} \text { charger location } \\ E & : \text { Energy, } \mathrm{kWh} \\ \mathrm{EV} & : \text { Electric vehicle } \\ g & : \text { Gravity }=9.8 \mathrm{~m}^{2} / \mathrm{s} \\ \mathrm{GHG} & : \text { Greenhouse gas } \\ \mathrm{GPS} & : \text { Global positioning system } \\ \mathrm{KAT} & : \text { Knoxville Area Transit } \\ \text { LHV } & : \text { Low heating value, } \mathrm{kJ} / \mathrm{kg} \\ m & : \text { Mass, } \mathrm{kg} \\ \dot{m} & : \text { Mass rate, } \mathrm{kg} / \mathrm{s} \\ \text { MAN } & : \text { Manhattan bus cycle } \\ \text { MD } & : \text { Medium-duty } \\ \text { NYBC } & : \text { New York bus cycle } \\ \text { OCTA } & : \text { Orange County Transit Authority }\end{array}$




\section{Submitted to Energy -An International Journal}

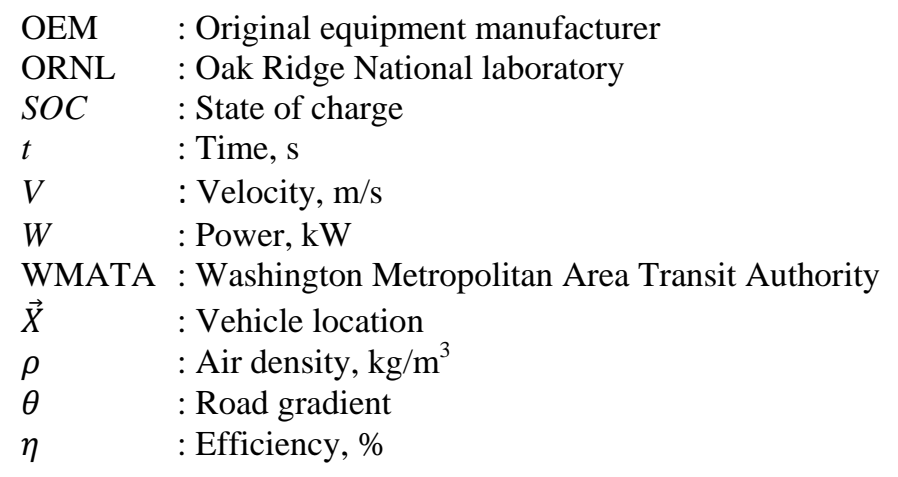

\section{Superscript:}

$\begin{array}{ll}e & : \text { Electric } \\ c & : \text { Conventional } \\ p & : \text { Peak }\end{array}$

\section{Subscript:}

$\begin{array}{ll}\text { Acc } & \text { : Accessory } \\ \text { batt } & \text { : Battery } \\ \text { chg } & \text {; Battery charge } \\ \text { chgr } & : \text { Charger } \\ c l & : \text { Clutch } \\ \text { dis } & \text { : Battery discharge } \\ \text { eng } & \text { : Engine } \\ f & \text { : Front or fuel } \\ f d & \text { : Final drive } \\ \text { gb } & \text { : Gearbox } \\ \text { mot } & \text { : Motor } \\ n c & \text { : Night charging } \\ \text { orc } & \text { : On-route charging } \\ \text { tract } & : \text { Tractive } \\ w h & : \text { Wheel }\end{array}$

\section{References}

1. Gao, Z., LaClair, T., Daw, C., Smith, D., et al., 2014. Simulations of the fuel economy and emissions of hybrid transit buses over planned local routes. SAE International Journal of Commercial Vehicles 7 (1), 216-237. DOI: 10.4271/2014-01-1562.

2. Altair. http://resources.altair.com/pdd/images/en-US/CaseStudy/BUSolutions-Developing-the-Worlds-First-Series-HydraulicHybrid-Bus.pdf. Accessed by February 21, 2016.

3. Noel, L., McCormack, R., 2014. A cost benefit analysis of a V2G-capable electric school bus compared to a traditional diesel school bus. Applied Energy, 126, 246-265.

4. Peak demand charges and electric transit buses.

http://www.calstart.org/Libraries/Publications/Peak_Demand_Charges_and_Electric_Transit_Buses_White_Paper.sflb.ashx. Accessed March 8, 2016.

5. ZEPS technology. http://zepsdrive.com/technology/. Accessed March 8, 2016.

6. DesignLine eco-smart I. http://cptdb.ca/wiki/index.php?title=DesignLine_Eco-Smart_I. Accessed March 8, 2016.

7. The future of transportation. http://www.proterra.com/product-tech/product-portfolio/\#charging. Accessed March 8, 2016.

8. Hengtong ebus. http://en.htebus.com/products_detail/\&productId=462e8b04-22b5-4f5e-b13d-71d9686a7232.html. Accessed March 8, 2016.

9. PRIMOVE for e-buses. http://primove.bombardier.com/applications/e-bus.html. Accessed March 8, 2016. 


\section{Submitted to Energy -An International Journal}

10. New flyer electric bus. http://www.newflyer.com/index/cms-filesystemaction/news\%20center/brochures/2014/electric\%20bus\%202014_4pager.pdf. Accessed March 8, 2016.

11. Pioneering clean technologies. http://www.balqon.com/wp-content/uploads/2013/09/m100_brochure_2013.pdf. Accessed March 8,2016

12. Electrify any truck. http://motivps.com/wp-content/uploads/2014/08/Commercial-TruckShuttleBus_1sheet_081214.pdf. Accessed March 8, 2016.

13. Barbosa, F., 2014. Pure electric bus traction technology overview - a path towards enhanced environmental performance and efficiency for transit bus fleets. SAE Technical Paper 2014-36-0205. DOI: 10.4271/2014-36-0205.

14. Hung, Y., Li, H., Lee, H., Chen, C. et al., 2013. System design and control strategy for a battery/supercapacitor electric bus with an integrated fast charger/bus stop station. SAE Technical Paper 2013-01-1459. DOI: 10.4271/2013-01-1459.

15. Higashi, H., 2015. Development of energy management for small electric buses. SAE Technical Paper 2015-01-0246. DOI: $10.4271 / 2015-01-0246$.

16. Korsesthakarn, K., Sripakagorn, A., 2014. Implementation of energy storage system with fleet management on electric shuttle buses. Energy Procedia 61, 1929-1932.

17. Perrotta, D., Ribeiro, B., Rossetti, R. J., Afonso, J.L., 2012. On the potential of regenerative braking of electric buses as a function of their itinerary. Procedia-Social and Behavioral Sciences 54, 1156-1167.

18. Lin, Z., 2014. Optimizing and diversifying electric vehicle driving range for US drivers. Transportation Science 48(4), 635-650.

19. Nurhadi, L., Borén, S., Ny, H., 2014. A sensitivity analysis of total cost of ownership for electric public bus transport systems in Swedish medium sized cities. Transportation Research Procedia 3, 818-827.

20. Lajunen, A., 2014. Energy consumption and cost-benefit analysis of hybrid and electric city buses. Transportation Research Part C: Emerging Technologies 38, 1-15.

21. Kühne, R., 2010. Electric buses-an energy efficient urban transportation means. Energy 35(12), $4510-4513$.

22. Miles, J., Potter, S., 2014. Developing a viable electric bus service: the Milton Keynes demonstration project. Research in Transportation Economics 48, 357-363.

23. Kontou, A., Miles, J., 2015. Electric buses: lessons to be learnt from the Milton Keynes demonstration project. Procedia Engineering 118, 1137-1144.

24. Zhou, B., Wu, Y., Zhou, B., Wang, R., et. al., 2016. Real-world performance of battery electric buses and their life-cycle benefits with respect to energy consumption and carbon dioxide emissions. Energy 96, 603-613.

25. LaClair, T., 2012. Application of a tractive energy analysis to quantify the benefits of advanced efficiency technologies for medium- and heavy-duty trucks using characteristic drive cycle data. SAE International Journal of Commercial Vehicles 5(1), 141-163. DOI: 10.4271/2012-01-0361.

26. Gao, Z., Smith, D.E., Daw, C.S., Edwards, K.D., et al., 2015. The evaluation of developing vehicle technologies on the fuel economy of long-haul trucks. Energy Conversion and Management 106, 766-781. DOI: 10.1016/j.enconman.2015.10.006.

27. Truck and bus charging standards discussion meeting. http://www.epri.com/Documents/Infrastructure\%20Working\%20Council\%20Meeting/1506/Truck\%20and\%20Bus\%20Meeting\% 20Presentations.pdf. Accessed February 22, 2016.

28. Lascurain, M.B., Franzese, O., Capps, G., Siekmann, A., et al., 2012. Medium Truck Duty Cycle Data from Real-World Driving Environments: Project Final Report. Technical Report, ORNL/TM-2012/240, Oak Ridge National Laboratory, Oak Ridge, Tennessee.

29. Discover safe, fast charging electric public transport. http://heliox.nl/wp-content/uploads/2014/06/Heliox-Fast-OpportunityCharge-120kW-200kW-500kW.pdf. Accessed March 8, 2016.

30. Autonomie. http://www.autonomie.net/. Accessed March 8, 2016.

31. Gao, Z., Finney, C., Daw, C., LaClair, T. et al., 2014. Comparative study of hybrid powertrains on fuel saving, emissions, and component energy loss in HD trucks. SAE International Journal of Commercial Vehicles 7(2), 414-431. DOI: 10.4271/2014-012326. 


\section{Submitted to Energy -An International Journal}

32. Karbowski, D., Delorme, A., Rousseau, A., 2010. Modeling the hybridization of a class 8 line-haul truck. SAE Technical Paper 2010-01-1931. DOI: 10.4271/2010-01-1931.

33. Gao, Z., Daw, C., Smith, D. LaClair, T. et al., 2016. Comparison of parallel and series hybrid powertrains for transit bus application. Transportation Research Record: Journal of the Transportation Research Board 2570, 97-106. DOI: 10.3141/2570-11.

34. Gao, Z., LaClair, T.J., Smith, D.E., Daw. C.S., 2015. Exploring fuel-saving potential of long-haul truck hybridization. Transportation Research Record: Journal of the Transportation Research Board 2502, 99-107. DOI: 10.3141/2502-12.

35. DOE Vehicle Technologies program, Multi-Year Program Plan 2011 - 2015. http://www1.eere.energy.gov/vehiclesandfuels/pdfs/program/vt_mypp_2011-2015.pdf. Accessed March 8, 2016.

36. Automotive System Cost Modeling Tool (ASCM). http://web.ornl.gov/sci/ees/etsd/cta/Auto\%20System\%20Cost\%20Modeling\%20Tool.pdf. Accessed March 8, 2016.

37. Gao, Z., Curran, S.J., Parks, J.E., Smith, D.E., et al., 2015. Drive cycle simulation of high efficiency combustions on fuel economy and exhaust properties in light-duty vehicles. Applied Energy 157, 762-776.

38. Measuring EV charging efficiency. http://teslaliving.net/2014/07/07/measuring-ev-charging-efficiency/. Accessed March 8, 2016.

39. BU-401a: fast and ultra-fast chargers. http://batteryuniversity.com/learn/article/ultra_fast_chargers. Accessed November 30, 2016.

40. The 10 minute charge might be in reach (but can the batteries survey?). http://www.electricmotornews.com/wpcontent/uploads/2016/01/Battery-Charge-Report.pdf. Accessed November 30, 2016.

41. Shirk, M., Wishart, J. 2015. Effects of electric vehicle fast charging on battery life and vehicle performance. SAE Technical Paper 2015-01-1190. DOI: 10.4271/2015-01-1190.

42. Does quick charging hurt battery life? total miles are more important. http://www.greencarreports.com/news/1090954_doesquick-charging-hurt-battery-life-total-miles-are-more-important. Accessed November 30, 2016. 


\section{Submitted to Energy -An International Journal}

\section{Table list:}

Table 1: List of commercially available electric buses and their electrical power characteristics.

Table 2: Characteristics of selected bus drive cycles, including the KAT drive cycle.

Table 3: Parameters used for simulating conventional and electric buses. 


\section{Submitted to Energy -An International Journal}

Table 1: List of commercially available electric buses and their electrical power characteristics.

\begin{tabular}{|c|c|c|c|c|}
\hline Vehicle & OEM & $\begin{array}{c}\text { Max motor } \\
\text { power }(\mathbf{k W})\end{array}$ & $\begin{array}{c}\text { Battery } \\
\text { Capacity (kWh) }\end{array}$ & $\begin{array}{c}\text { Charger power } \\
\text { (kW) }\end{array}$ \\
\hline 40-60 ft Transit bus[4] & BYD & $180-360$ & $324-548$ & $40 / 80 / 100 / 200$ \\
\hline 30-40ft Transit bus [5] & CCW & - & 311 & 40 \\
\hline 34-40ft Transit bus[6] & Designline & 335 & 261.8 & - \\
\hline 35-40ft Transit bus[7] & Proterra & 220 & $53-321$ & 500 \\
\hline 40 ft Transit bus [4] & EBusco & - & $242-311$ & 125 \\
\hline 40 ft Transit bus [8] & Hengtong & 180 & $61-78$ & 400 \\
\hline 40 ft Transit bus [9] & PRIMOVE & $200-400$ & $60-90$ & 200 \\
\hline 40 ft Transit bus [4,10] & New Flyer & 160 & $200-300$ & 500 \\
\hline Shuttle [11] & Balqon & 168 & 312 & $40 / 100$ \\
\hline Shuttle [12] & Motiv & 150 & $80-120$ & 60 \\
\hline Trolleybus [4] & ABB & - & 38 & $40 / 200 / 400$ \\
\hline
\end{tabular}

$1 \mathrm{ft}=0.305 \mathrm{~m}$. 
Submitted to Energy -An International Journal

Table 2: Characteristics of selected bus drive cycles, including the KAT drive cycle.

\begin{tabular}{|l|c|c|c|c|c|}
\hline Cycle & KAT & MAN & NYBC & OCTA & WMATA \\
\hline Duration (s† or hours*) & $4,714 *$ & $2,178 \dagger$ & $620 \dagger$ & $1,909 \dagger$ & $1,839 \dagger$ \\
\hline Distance $(\mathrm{km})$ & 71,479 & 6.65 & 0.98 & 10.53 & 6.86 \\
\hline Average Speed $(\mathrm{km} / \mathrm{hr})$ & 15.13 & 11.00 & 5.75 & 19.85 & 13.42 \\
\hline Maximum Speed $(\mathrm{km} / \mathrm{hr})$ & 111.25 & 40.73 & 49.43 & 65.41 & 76.48 \\
\hline Percent of Idle Time & $52.4 \%$ & $39.2 \%$ & $68.9 \%$ & $26.9 \%$ & $43.8 \%$ \\
\hline Maximum Acceleration $\left(\mathrm{m} / \mathrm{s}^{2}\right)$ & 5.3 & 4.60 & 6.10 & 4.06 & 3.00 \\
\hline Maximum Deceleration $\left(\mathrm{m} / \mathrm{s}^{2}\right)$ & -7.6 & -5.60 & -4.30 & -5.13 & -4.50 \\
\hline
\end{tabular}


Table 3: Parameters used for simulating conventional and electric buses.

\begin{tabular}{|c|c|c|}
\hline Vehicle Parameters & Conventional & Electric \\
\hline Frontal area $\left(\mathbf{m}^{\mathbf{2}}\right) \boldsymbol{A}_{\mathbf{f}}$ & 8.5 & 8.5 \\
\hline Rolling resistance coefficient $C_{r r}$ & 0.0098 & 0.0098 \\
\hline Aerodynamic drag coefficient $C_{d}$ & 0.79 & 0.79 \\
\hline Bus mass $(\mathbf{k g}) \boldsymbol{m}$ & $10,207-13,636$ & $10,437-15,626$ \\
\hline Engine efficiency $\eta_{\boldsymbol{e n g}}$ & Eq. $3(\mathrm{~b})$ & - \\
\hline Clutch efficiency $\eta_{c l}$ & 0.86 & - \\
\hline Gearbox efficiency $\eta_{g b}$ & 0.92 & - \\
\hline Final Drive efficiency $\eta_{f d}$ & 0.98 & 0.98 \\
\hline Wheel drive efficiency $\eta_{w h}$ & 0.99 & 0.99 \\
\hline Motor efficiency $\eta_{m o t}$ & - & 0.88 \\
\hline Battery efficiency $\eta_{b a t t}$ & - & 0.98 \\
\hline EV Battery Capacity $(\mathbf{k W h}) E_{b a t t}$ & - & $60-324$ \\
\hline Charger efficiency $\eta_{c h g r}$ & - & $0.97[31]$ \\
\hline Accessories $(\mathbf{k W}) W_{a c c}$ & 7.5 & 3.75 \\
\hline
\end{tabular}




\section{Submitted to Energy -An International Journal}

\section{Figure List:}

Figure 1: The framework tool for bus electrification feasibility studies

Figure 2: One-year routes of transit bus coverage in Knoxville, TN. The red and blue dots are the bus depot and garage, respectively. The distance between these locations is $1.93 \mathrm{~km}$ (i.e. $1.2 \mathrm{mile}$ ).

Figure 3: Driving statistics of the three KAT transit city buses.

Figure 4: The validation of the engine energy consumption for the conventional transit bus (a) and the electric bus battery energy consumption (b) over the driving conditions of the selected two-hour real-world KAT bus data. The simulated conventional and electric bus weights were $11,636 \mathrm{~kg}$ and $13,626 \mathrm{~kg}$, respectively.

Figure 5: Energy consumption of conventional and electric buses as a function of the daily drive distance based on the full year of KAT real-world driving data. Each point represents one day. The simulated electric bus was powered by a $324 \mathrm{kWh}$ battery with $90 \mathrm{~kW}$ proactive charging and minimum 2 hours per charge.

Figure 6: Proactive recharging demand as a function of the daily drive distance and time based on the one-year KAT real-world driving data. Each point represents one complete day of driving. The simulated electric bus was powered by a $324 \mathrm{kWh}$ battery with $90 \mathrm{~kW}$ proactive charging and minimum 2 hours per charge.

Figure 7: Impact of battery size on the proactive charging and lost drive time based on one year of KAT real-world driving data. The simulated battery size covers 150-324 kWh while the proactive charging is $90 \mathrm{~kW}$ and lasts a minimum of 2 hours.

Figure 8: Effect of battery capacity and bus operational range on on-route charging demand and SOC performance over two typical daily operation scenarios. The simulated battery size covers $150-324 \mathrm{kWh}$, while the proactive charging is $90 \mathrm{~kW}$ and lasts for a minimum of 2 hours.

Figure 9: Effect of battery capacity on energy consumption, drive time and distance ranges for the four standardized drive cycles without any boost charging or proactive charging. In the simulation, the simulated battery SOC started at $100 \%$ and ended at $10 \%$.

Figure 10: Impact of battery size on proactive charging and missed drive time based on the one-year KAT real-world driving data. The minimum charging times for $90 \mathrm{~kW}$ and $480 \mathrm{~kW}$ chargers are 2 hours and a half hour, respectively.

Figure 11: Effect of battery size and bus operational range for on-route charging demand and SOC performance over two typical daily operational routes. The minimum charging times for $90 \mathrm{~kW}$ and $480 \mathrm{~kW}$ chargers are 2 hours and one-half hour, respectively. 




Figure 1: The framework tool for bus electrification feasibility studies 


\section{Submitted to Energy -An International Journal}



Figure 2: One-year routes of transit bus coverage in Knoxville, TN. The red and blue dots are the bus depot and garage, respectively. The distance between these locations is $1.93 \mathrm{~km}$ (i.e. 1.2 mile). 
Submitted to Energy -An International Journal
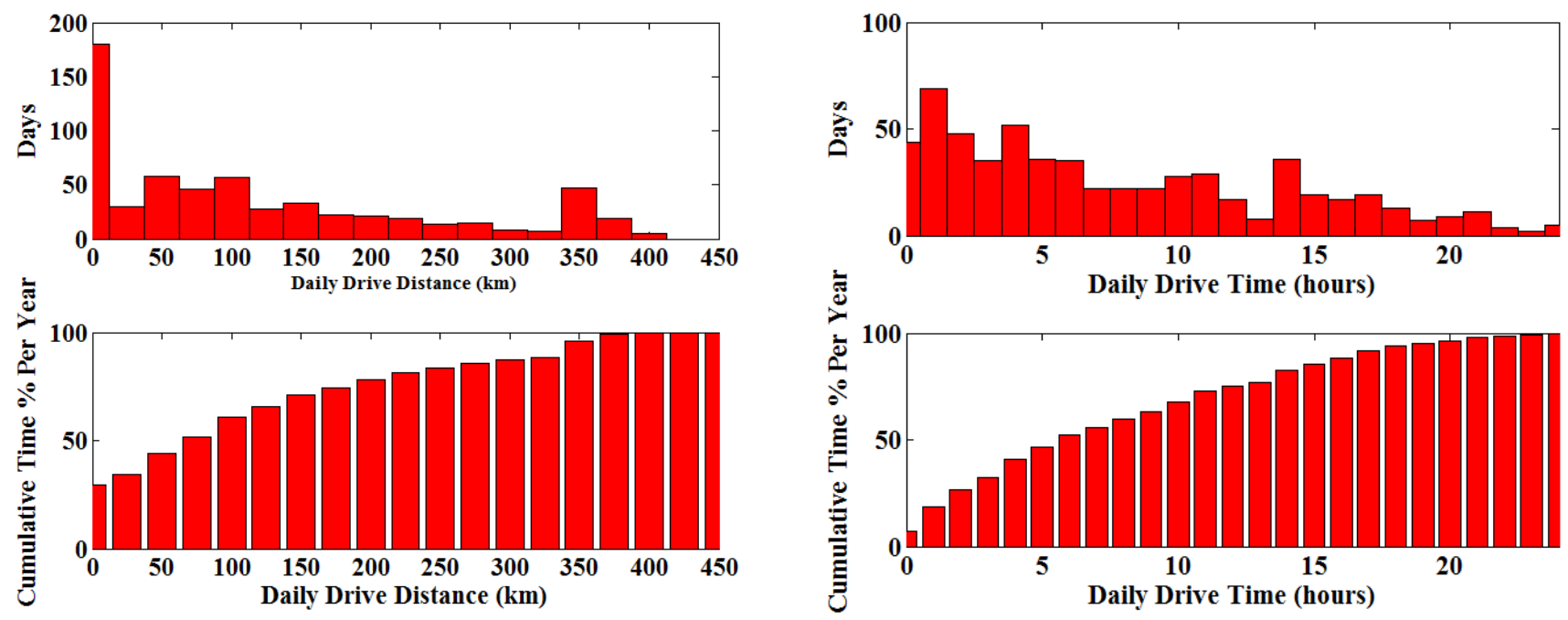

Figure 3: Driving statistics of the three KAT transit city buses. 


\section{Submitted to Energy -An International Journal}
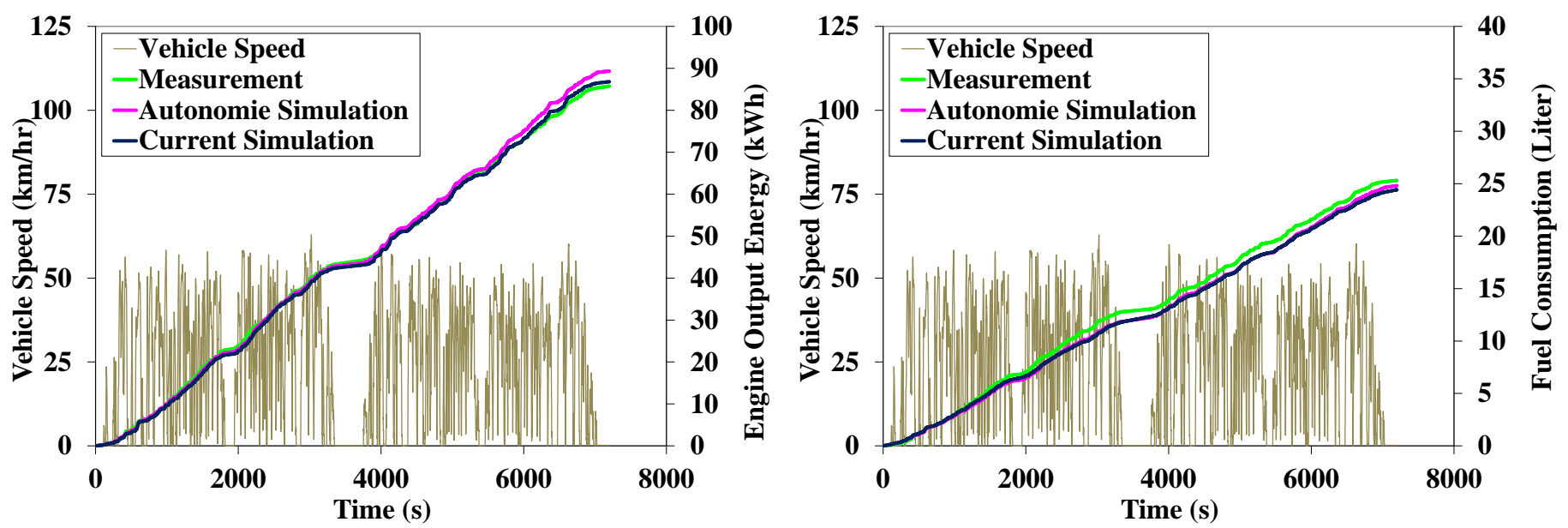

(a) Conventional vehicle

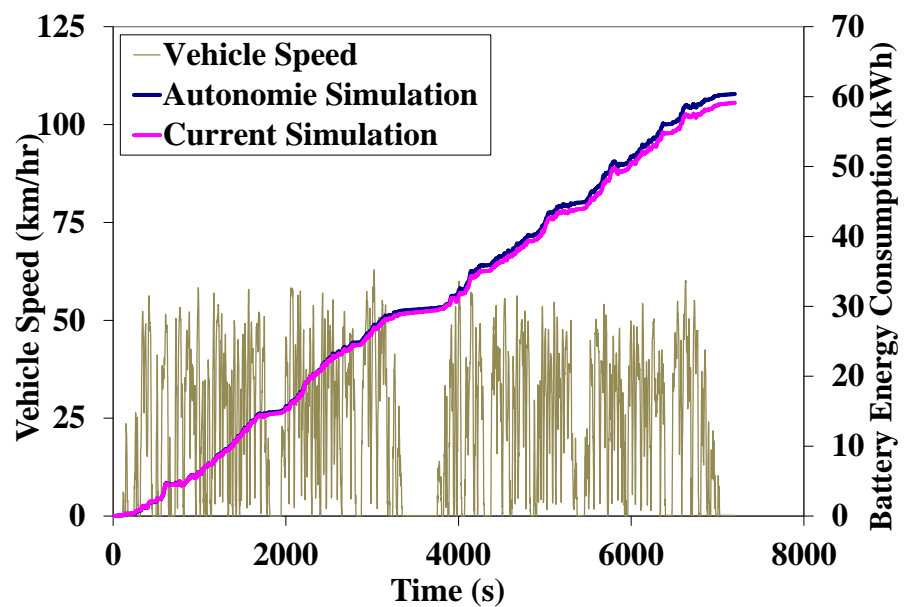

(b) Electric vehicle

Figure 4: The validation of the engine energy consumption for the conventional transit bus (a) and the electric bus battery energy consumption (b) over the driving conditions of the selected two-hour real-world KAT bus data. The simulated conventional and electric bus weights were $11,636 \mathrm{~kg}$ and $13,626 \mathrm{~kg}$, respectively. 


\section{Submitted to Energy -An International Journal}

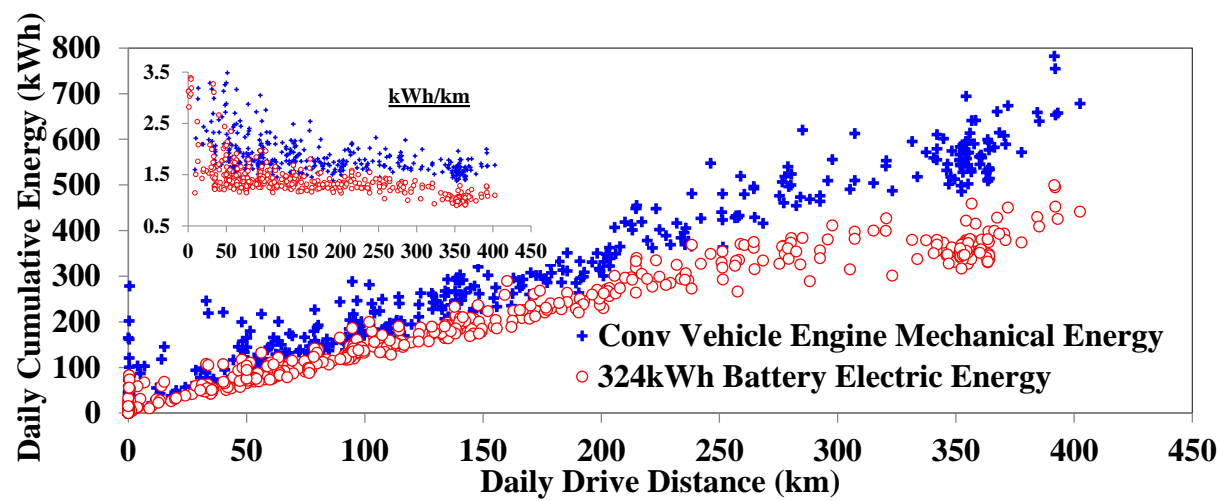

(a) Cumulative energy consumption

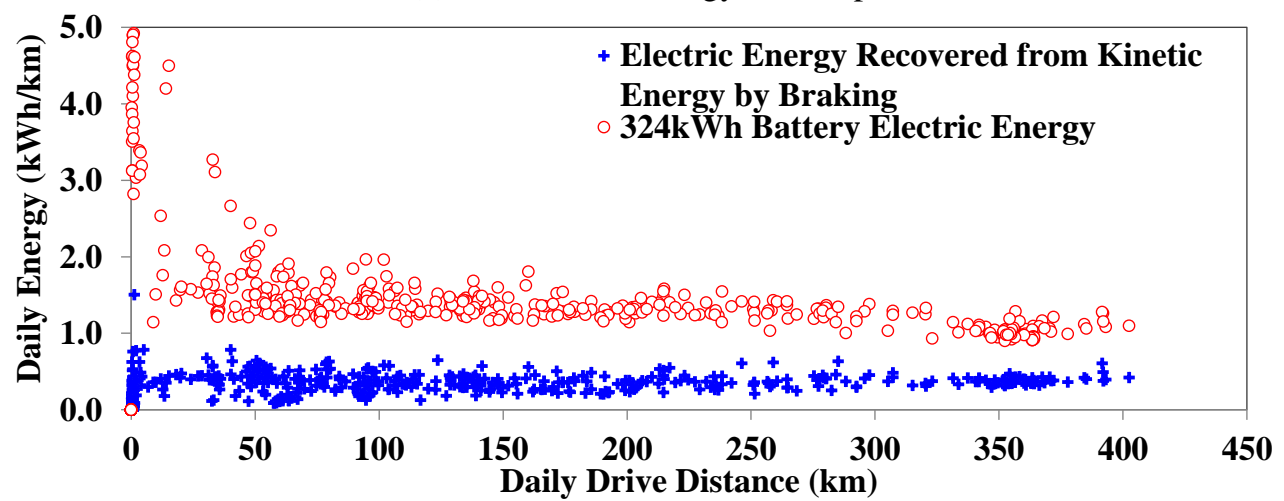

(b) Energy consumption per km

Figure 5: Energy consumption of conventional and electric buses as a function of the daily drive distance based on the full year of KAT real-world driving data. Each point represents one day. The simulated electric bus was powered by a $324 \mathrm{kWh}$ battery with 90 $\mathrm{kW}$ proactive charging and minimum 2 hours per charge. 


\section{Submitted to Energy -An International Journal}

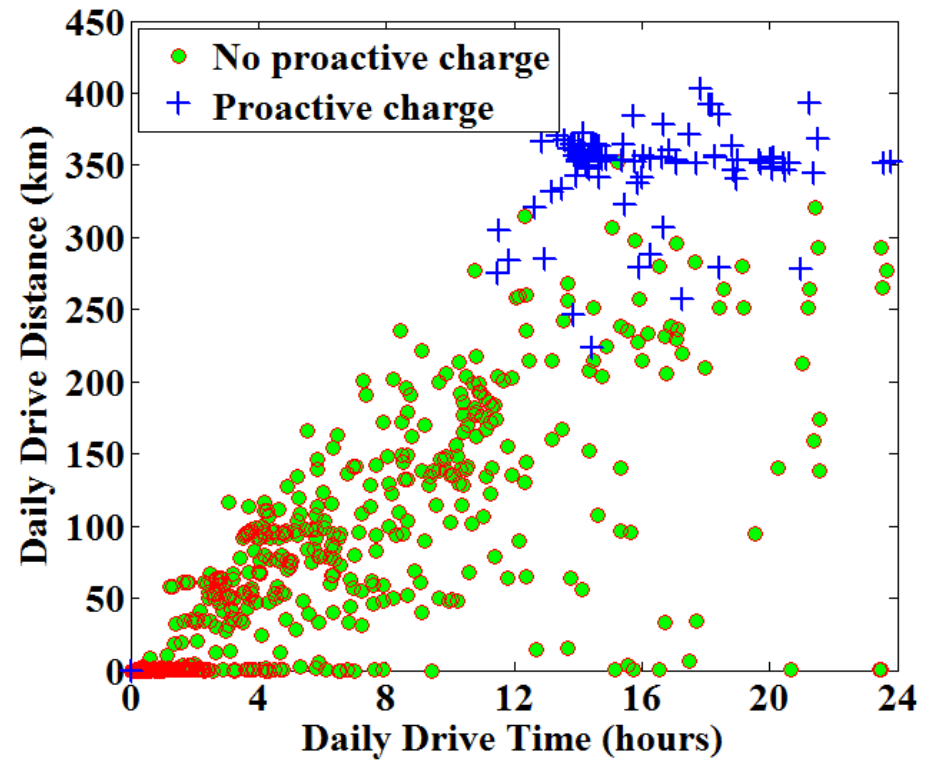

Figure 6: Proactive recharging demand as a function of the daily drive distance and time based on the one-year KAT real-world driving data. Each point represents one complete day of driving. The simulated electric bus was powered by a $324 \mathrm{kWh}$ battery with $90 \mathrm{~kW}$ proactive charging and minimum 2 hours per charge. 


\section{Submitted to Energy -An International Journal}

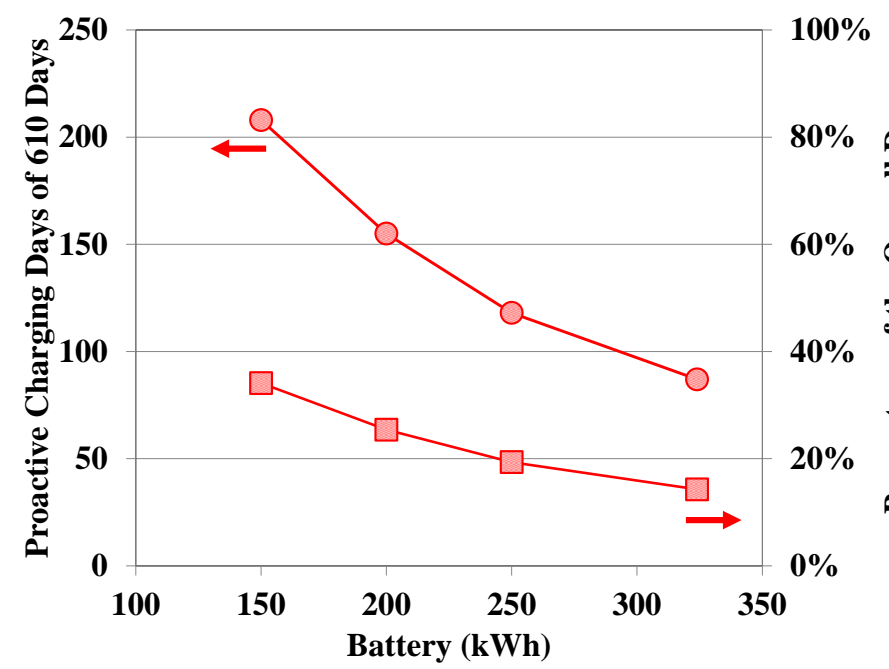

(a) Proactive charging days

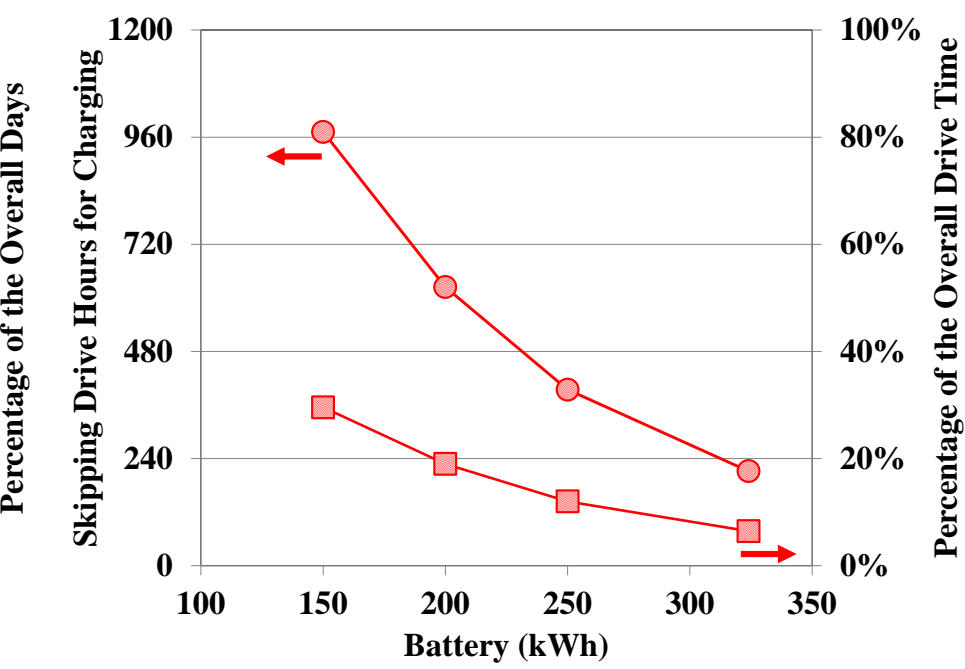

(b) Lost drive time for proactive charging

Figure 7: Impact of battery size on the proactive charging and lost drive time based on one year of KAT real-world driving data. The simulated battery size covers $150-324 \mathrm{kWh}$ while the proactive charging is $90 \mathrm{~kW}$ and lasts a minimum of 2 hours. 




(a) Short route with $20.9 \mathrm{~km}$ (i.e. 13 miles)

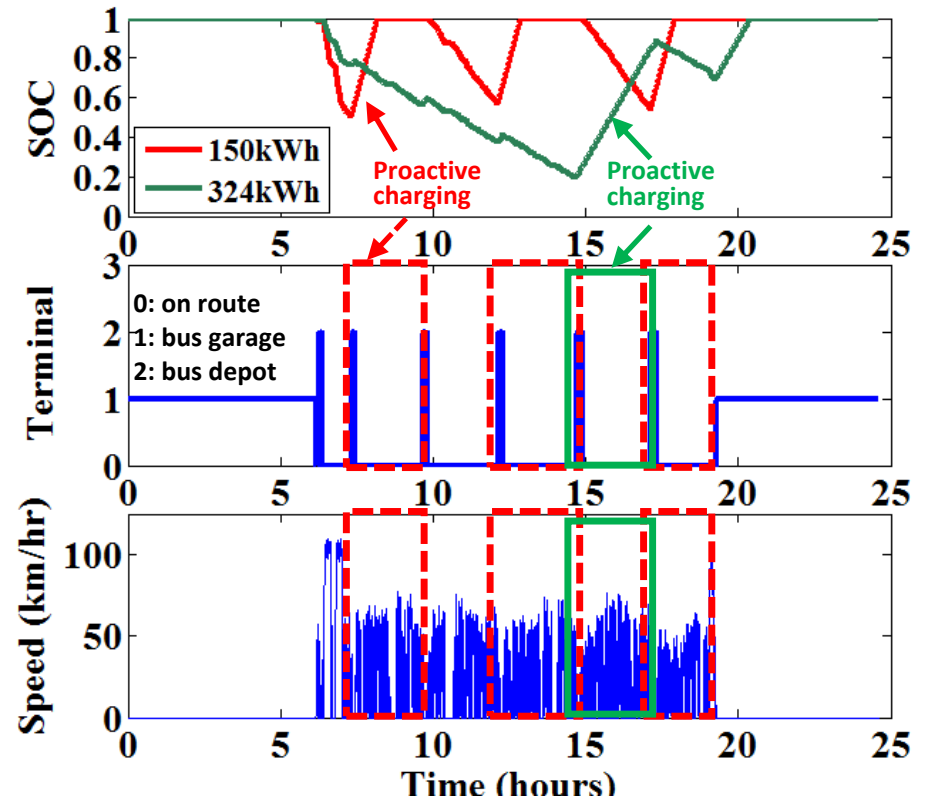

(b) Long route with $61.2 \mathrm{~km}$ (i.e. 38 miles)

Figure 8: Effect of battery capacity and bus operational range on on-route charging demand and SOC performance over two typical daily operation scenarios. The simulated battery size covers $150-324 \mathrm{kWh}$, while the proactive charging is $90 \mathrm{~kW}$ and lasts for a minimum of 2 hours. 


\section{Submitted to Energy -An International Journal}

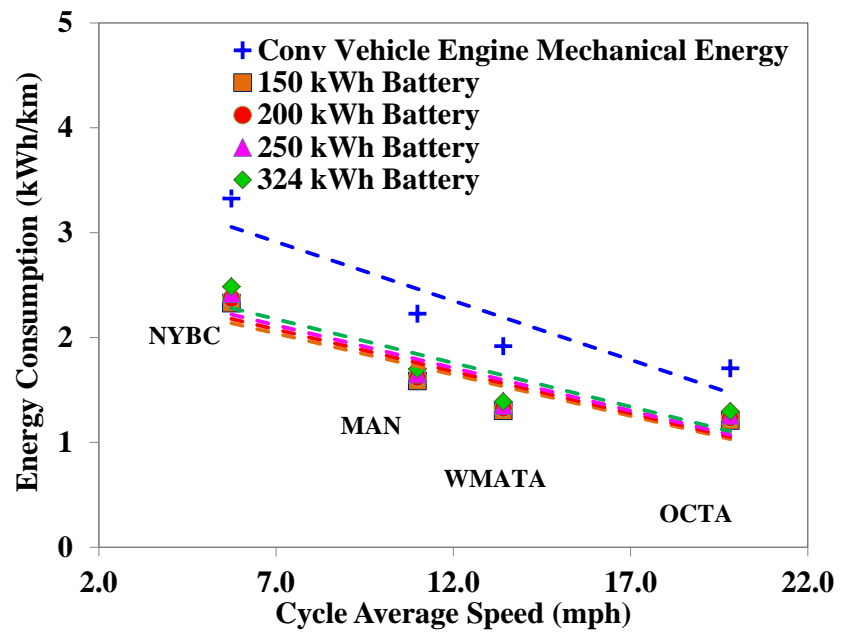

(a) Energy consumption per km

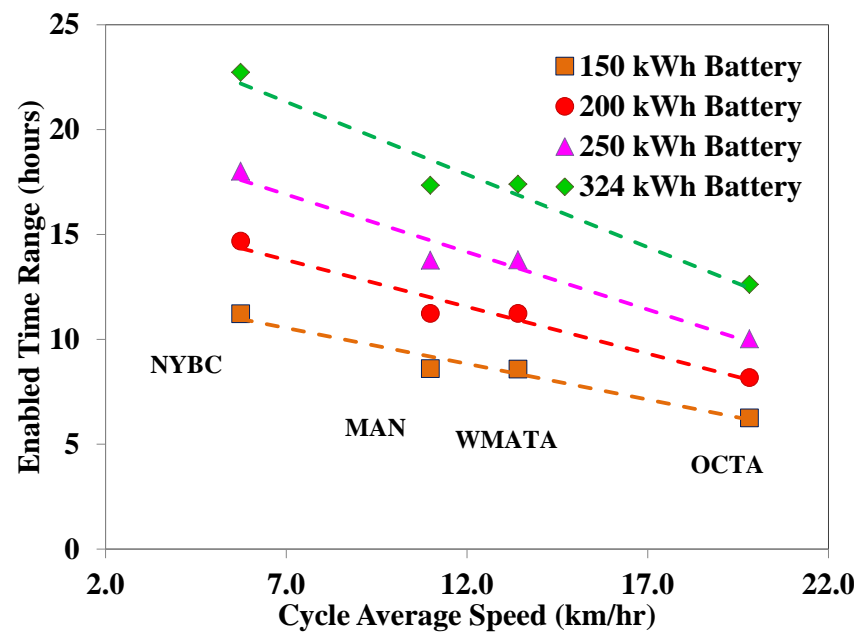

(b) Drive time

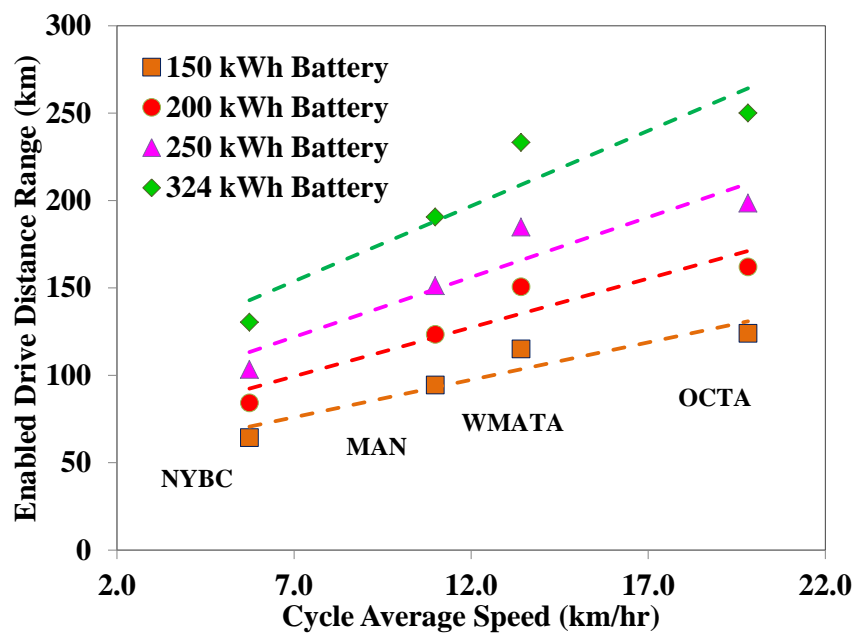

(c) Drive mileage

Figure 9: Effect of battery capacity on energy consumption, drive time and distance ranges for the four standardized drive cycles without any boost charging or proactive charging. In the simulation, the simulated battery SOC started at 100\% and ended at $10 \%$. 


\section{Submitted to Energy -An International Journal}

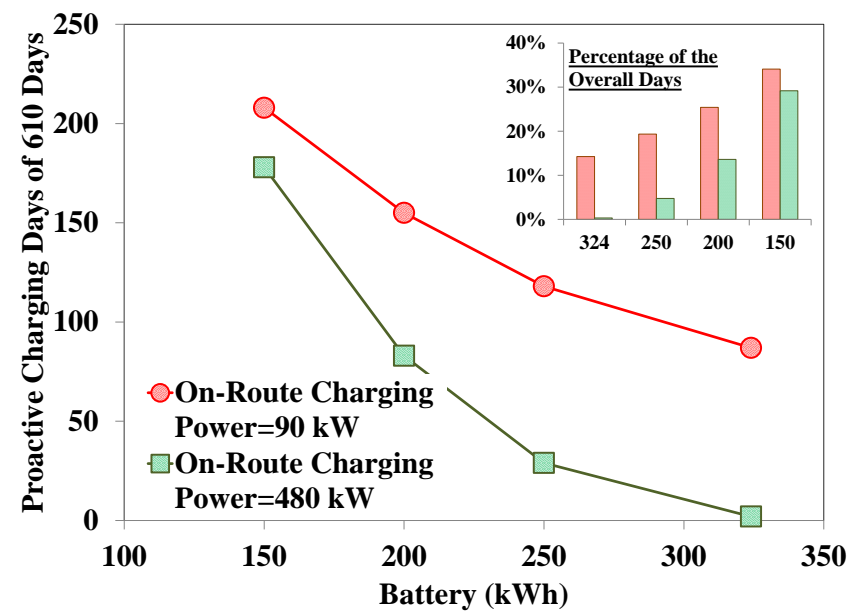

(a) Proactive charging days

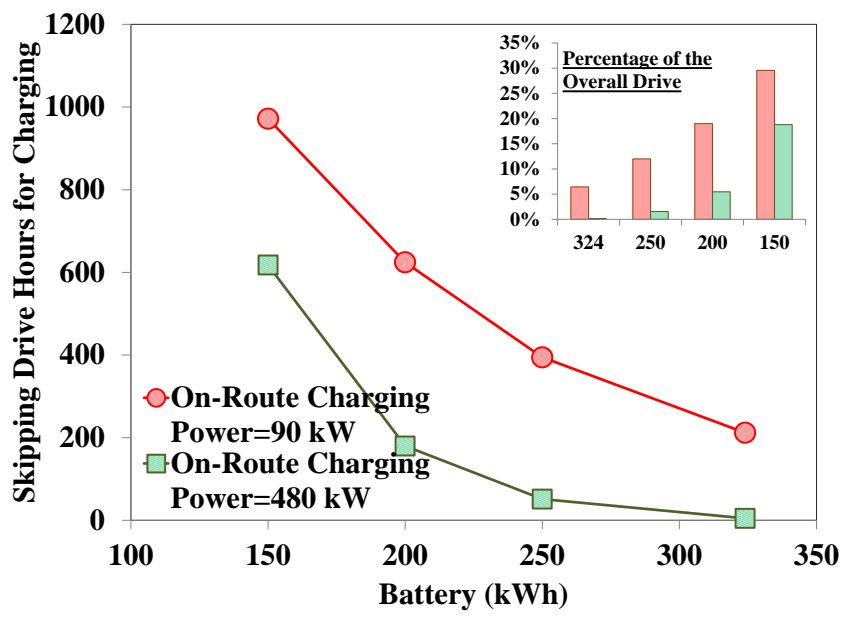

(b) Missed drive time for proactive charging

Figure 10: Impact of battery size on proactive charging and missed drive time based on the one-year KAT real-world driving data. The minimum charging times for $90 \mathrm{~kW}$ and $480 \mathrm{~kW}$ chargers are 2 hours and a half hour, respectively. 


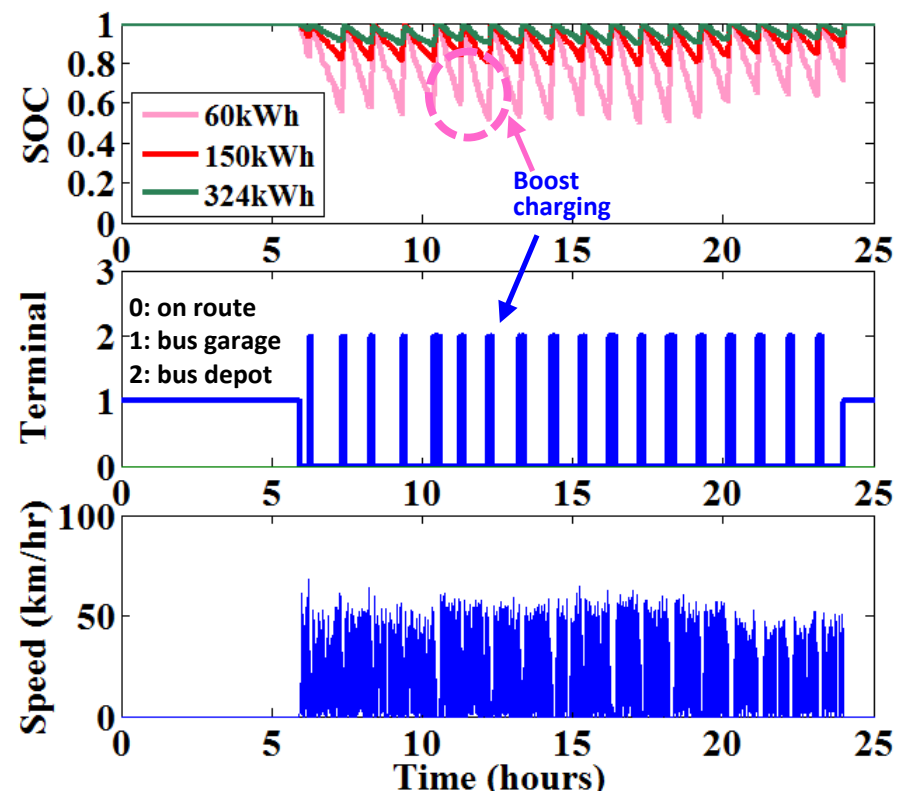

(a) Short route with $20.9 \mathrm{~km}$ (i.e. 13 miles)

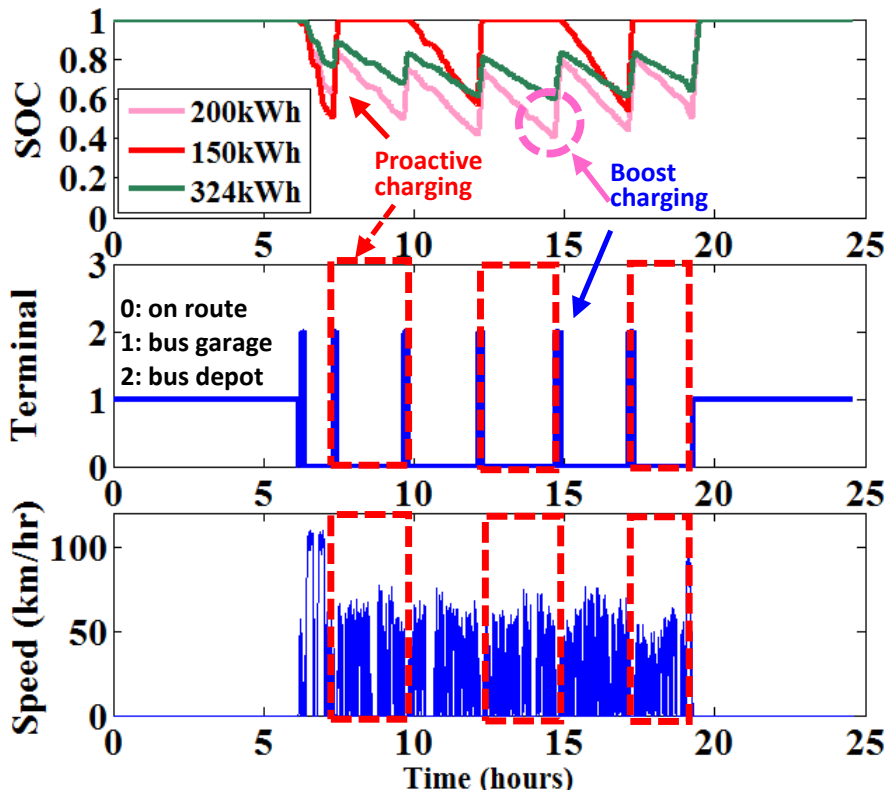

(b) Long route with $61.2 \mathrm{~km}$ (i.e. 38 miles)

Figure 11: Effect of battery size and bus operational range for on-route charging demand and SOC performance over two typical daily operational routes. The minimum charging times for $90 \mathrm{~kW}$ and $480 \mathrm{~kW}$ chargers are 2 hours and one-half hour, respectively. 